\title{
Gastric antral vascular ectasia in a pediatric patient with neuroblastoma who underwent tandem stem cell transplantation
}

\author{
Yumiko Sugishita, Shohei Yamamoto, Ryota Kaneko, Naoko Okamoto, Masaya Koganesawa, Sachio Fujita, \\ Kosuke Akiyama, Ryosuke Matsuno, Daisuke Toyama, Keiichi Isoyama
}

Department of Pediatrics, Showa University Fujigaoka Hospital, Japan

\begin{abstract}
Gastric antral vascular ectasia (GAVE) is an angiodysplastic disorder, which causes severe and prolonged gastric bleeding. Although GAVE has been described in adult patients treated with hematopoietic stem cell transplantation (HSCT), a few cases involving pediatric patients have also been reported. A 5-year-old boy with neuroblastoma (NB) developed severe hematemesis after undergoing tandem HSCT, i. e. autologous peripheral blood stem cell transplantation (auto-PBSCT), followed by allogeneic cord blood transplantation (allo-CBT). The patient suffered oral feeding difficulties because of the effects of chemotherapy and an unbalanced diet. Intravenous Busulfan (ivBU) was used as a conditioning regimen for the auto-PBSCT. The diagnosis of GAVE was made based on endoscopy of the upper gastrointestinal tract on day 31 after the allo-CBT. Argon plasma coagulation (APC) was performed twice, and the complete resolution of GAVE was confirmed by an endoscopic re-evaluation, conducted on day 87. GAVE in this case might have been associated with ivBU treatment. Atrophy of the gastric mucosa due to loss of appetite might also have contributed to GAVE. NB was treated using high-doses of alkylating agents, such as BU. Such treatment can cause significant mucositis of the oral cavity as well as vascular lesions and is associated with GAVE. Therefore, GAVE should be considered when gastrointestinal bleeding occurs in NB patients treated with HSCT. APC might be effective against HSCT-GAVE.
\end{abstract}

Key words: gastric antral vascular ectasia, neuroblastoma, busulfan, tandem SCT

Submitted August 24, 2018; Accepted October 2, 2018; Published online January 15, 2019; Issued online Feburuary 25, 2019

Correspondence: Yumiko Sugishita, Department of Pediatrics, Showa University Fujigaoka Hospital, 1-30 Fujigaoka, Aoba-ku, Yokohama 227-8501, Japan. E-mail: kohola55@gmail.com

\section{Introduction}

Gastric antral vascular ectasia (GAVE) is a rare disorder often causing severe bleeding in the upper gastrointestinal tract ${ }^{1}$. GAVE is sometimes observed after hematopoietic stem cell transplantation (HSCT), and the incidence of GAVE after HSCT (HSCT-GAVE) is reported to be about $2.2 \%^{2}$. Although, it has been found to be associated with the use of busulfan (BU) and sinusoidal obstruction syndrome $(\mathrm{SOS})^{3}$, the pathogenesis of HSCT-GAVE is still unclear. Here, we describe the case of a neuroblastoma (NB) patient with HSCT-GAVE, who underwent tandem HSCT.

\section{Case presentation}

A 5-year-old boy was admitted to our hospital due to loss of appetite. He was generally well apart from being anemic (hemoglobin level: $9.5 \mathrm{~g} / \mathrm{dl}$ ) and weighed 13.3 $\mathrm{kg}$ (standard deviation: - 2.1) on admission. Other investigations revealed a right adrenal tumor, and a biopsy examination demonstrated that it was a neuroblastoma. Multiple bone metastases and bone involvement were detected, which were comparable with stage 4 of the disease and the anemia could have been the result of bone marrow metastasis. The patient was treated with 5 courses of chemotherapy, followed by a high-dose chemotherapy and autologous peripheral blood stem cell transplantation (auto-PBSCT). After the chemotherapy, the patient underwent primary surgical resection and 


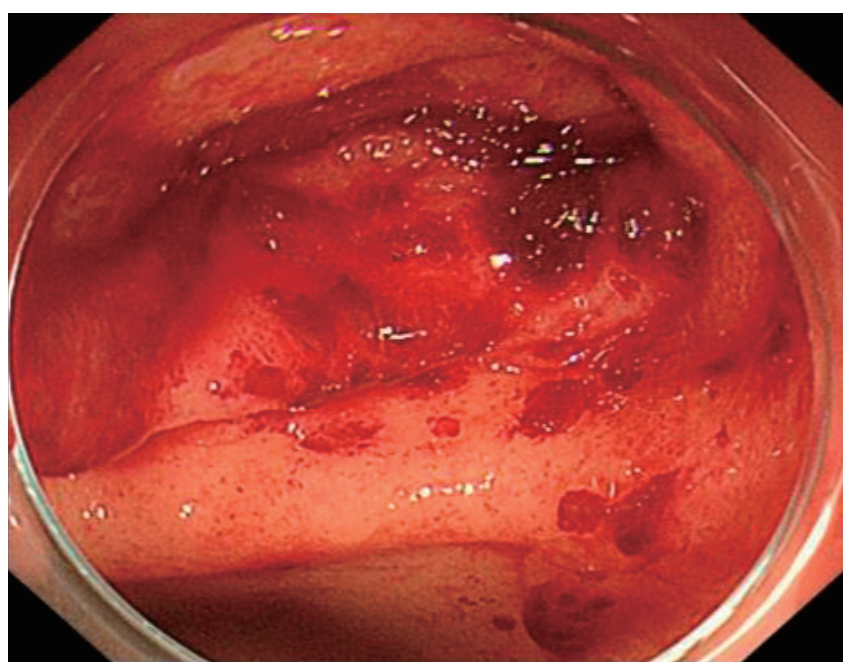

Figure 1. Endoscopic appearance of gastric antrum

An endoscopic image of the patient obtained on day 32 after the cord blood transplant.

Capillary enlargement and randomly distributed red spots were seen in the antrum (so-called "honeycomb stomach").

radiotherapy (primary tumor: $30.6 \mathrm{~Gy}$ and metastatic lesions: $19.8 \mathrm{~Gy}$ ). The PBSCT conditioning regimen consisted of intravenous BU (ivBU) $(19.2 \mathrm{mg} / \mathrm{kg})$ and melphalan $(\mathrm{L}-\mathrm{PAM})\left(180 \mathrm{mg} / \mathrm{m}^{2}\right)$. All of these therapies were well tolerated. However, due to the effects of chemotherapy and an unbalanced diet, the patient experienced oral feeding difficulties during the course of chemotherapy. Since the patient did not achieve a complete remission, he had to undergo allogeneic cord blood transplantation (allo-CBT) 4 months after the auto-PBSCT. The conditioning regimen consisted of L-PAM $(140 \mathrm{mg}$ / $\mathrm{m}^{2}$ ), fludarabine $\left(125 \mathrm{mg} / \mathrm{m}^{2}\right)$, and Total Body Irradiation (TBI) (2 Gy). The prophylaxis for GVHD consisted of tacrolimus and short-term methotrexate. Tarry stools were observed from day 21 . Sudden occurrence of hematemesis was observed on day 31 , and the patient's hemoglobin level decreased from 10 to $5.2 \mathrm{~g} / \mathrm{dl}$. Endoscopy of the upper gastrointestinal tract revealed enlargement of the capillaries and randomly distributed red spots in the antrum, which were indicative of GAVE (Figure 1). APC therapy was performed twice, on days 31 and 49. Both fat and albumin formulas were required to restore the gastrointestinal mucous membrane. The patient's tarry stools improved after day 75 , and the complete resolution of GAVE was confirmed during an endoscopic re-evaluation conducted on day 87. Oral food consumption resumed at this time. The patient was well and had not suffered recurrent GAVE or NB at 24 months after the CBT.

\section{Discussion}

HSCT-GAVE was first recognized as a cause of gastro- intestinal bleeding in $1994^{4}$. Male gender, oral BU as a part of the conditioning regimens, and SOS are the factors associated with the development of HSCT-GAVE ${ }^{3}$. Hirayama et al. summarized the cases of 30 patients with HSCT-GAVE, oral BU (poBU)-based conditioning therapies were used in all case studies ${ }^{5}$. High-dose of BU can cause significant mucositis of the oral cavity ${ }^{6}$ and may also cause direct chemical damage to the gastric mucosa ${ }^{3,4}$. Although our patient received ivBU, Fukuda et al. reported two cases of HSCT-GAVE involving patients who had been treated with ivBU containing conditioning regimens rather than $\mathrm{poBU}^{7}$. Another possible pathogenic mechanism for GAVE could be age-associated ischemic or degenerative changes in the gastric mucosa ${ }^{8}$. In addition to ivBU, atrophy of gastric mucosa due to loss of appetite might have contributed to GAVE. Interestingly, our patient developed HSCT-GAVE after CBT rather than after auto-PBSCT wherein ivBU was used as a conditioning regimen. Analysis of the summarized data indicated that around $25 \%$ of patients developed HSCT-GAVE post-transplantation after day $150^{9}$. In a previous study, two patients who received ivBU developed HSCT-GAVE on post-transplantation days 84 and $145^{7}$. Our patient developed HSCT-GAVE on day 153 after the first transplantation (auto-PBSCT) procedure.

To our knowledge, only two pediatric cases (EpsteinBarr virus-associated hemophagocytic lymphohistiocytosis [EBV-HLH] and acute myelogenous leukemia $[\mathrm{AML}]$ ) of HSCT-GAVE have been reported ${ }^{5,9}$. poBUcontaining conditioning regimens were used in both of these patients. They did suffer grade I acute GVHD and limited chronic GVHD, respectively. The AML patient developed concomitant protein-losing enteropathy and 
HSCT-GAVE and was successfully treated with cyclosporin A and prednisolone ${ }^{5}$. Our patient did not show evidence of chronic GVHD at the onset of GAVE. However, Grant et al. described the association between HSCT-GAVE and chronic GVHD ${ }^{10}$. There seems to be an immune-mediated link between chronic GVHD and the development of HSCT-GAVE that is supported by a similar development of GAVE in patients with systemic sclerosis $^{10}$. The EBV-HLH patient was successfully treated with endoscopic coagulation therapy ${ }^{9}$. Our patient was successfully treated with two rounds of APC. Although, there is no established therapy for HSCT-GAVE, endoscopic treatment has been used in many cases ${ }^{3}$. Fukuda et al. reported that $A P C$ was effective against HSCTGAVE $^{7}$. APC might be a feasible treatment for HSCTGAVE because it is effective and easy to perform, even in pediatric patients.

\section{Acknowledgement}

The authors thank Dr. Yuichiro Kuroki who underwent endoscopy of the upper gastrointestinal tract and APC.

\section{Authors' Contributions}

Y. S. and S. Y. designed and performed the research and wrote the paper, and R. K., N. O., M. K., S. F., K. A., R. M., D. T., and K. I. collected and managed the clinical data.

\section{Conflict of Interest}

The authors declare that they have no conflicts of interest. Disclosure forms provided by the authors are available here.

\section{References}

1. Dulai GS, Jensen DM, Kovacs TO, Gralnek IM, Jutabha R. Endoscopic treatment outcomes in watermelon stomach patients with and without portal hypertension. Endoscopy. 2004; 36: 68-72

2. Ohashi K, Sanaka M, Tu Y, Egawa N, Ohashi K, Funata N, et al. Clinical features and treatment of hematopoietic stem cell transplantation-associated gastric antral vascular ectasia. Bone Marrow Transplant. 2003; 32: 417-21.

3. Tobin RW, Hackman RC, Kimmey MB, Durtschi MB, Hayashi A, Malik R, et al. Bleeding from gastric antral vascular ectasia in marrow transplant patients. Gastrointest Endosc. 1996; 44: 223-9.

4. Marmaduke DP, Greenson JK, Cunningham I, Herderick EE, Cornhill JF. Gastric vascular ectasia in patients undergoing bone marrow transplantation. Am J Clin Pathol. 1994; 102: 194-8.

5. Hirayama M, Azuma E, Nakazawa A, Iwamoto S, Toyoda H, Komada Y. Simultaneous occurrence of gastric antral vascular ectasia and protein-losing enteropathy in chronic graft-versushostdisease. Int J Hematol. 2013; 97: 529-34.

6. Buggia I, Locatelli F, Regazzi MB, Zecca M. Busulfan. Ann Pharmacother. 1994; 28: 1055-62.

7. Fukuda K, Kurita N, Sakamoto T, Nishikii H, Okoshi Y, Sugano $\mathrm{M}$, et al. Post-transplant gastric antral vascular ectasia after intra-venous busulfan regimen. Int J Hematol. 2013; 98: 135-8.

8. Lee FI, Costello F, Flanagan N, Vasudev KS. Diffuse antral vascular ectasia. Gastrointest Endosc. 1984; 30: 87-90

9. Kuroiwa Y, SuzukiN, Mizue N, Hori T, Endo T, Yoshida Y, et al. Gastric antral vascular ectasia in 2-yr-old girl undergoing unrelated cord blood stem cell transplantation. Pediatr Transplant. 2005; 9: 788-91

10. Grant MJ, Horwitz ME. HSCT-GAVE as a manifestation of chronic graft versus host disease: A case report and review of the existing literature. Case Rep Transplant. 2018; 2018: 2376483.

https: //doi.org/10.31547/bct-2018-007

Copyright (C) 2018 APBMT. All Rights Reserved. 